\title{
ESTUDO DA VARIABILIDADE DA RETENÇÃO DO PRESERVANTE CCA TIPO A NA MADEIRA DE Brosimum rubescens Taub. MORACEAE - (PAU-RAINHA) UMA ESPÉCIE MADEIREIRA DA REGIÃO AMAZÔNICA ${ }^{1}$
}

\author{
Ceci Sales-Campos², Basilio Frasco Vianez² e Maria Silvia de Mendonça ${ }^{3}$
}

\begin{abstract}
RESUMO - Avaliou-se a variabilidade da tratabilidade da espécie madeireira Brosimum rubescens Taub. Moraceae (pau-rainha), abordando três diferentes alturas do tronco (base, meio e ápice) e duas partes (cerne e alburno). O processo utilizado foi o de impregnação sob pressão, através do método de célula-cheia ou Bethell, e o preservante foi o CCA, tipo A, a $2 \%$ de concentração. A análise da variabilidade deu-se em função da retenção. Ao comparar os dados de retenção $\left(\mathrm{kg} / \mathrm{m}^{3}\right)$ das diferentes alturas e partes do tronco, constatou-se que o grau de tratabilidade do alburno é moderadamente difícil, enquanto o cerne é refratário. Os resultados de retenção das toras estudadas nas diferentes alturas da árvore não apresentaram diferença significativa, entretanto existe diferença significativa de retenção entre as partes (cerne e alburno). A análise de microdistribuição do preservante através do estudo anatômico funciona como ferramenta auxiliar na interpretação desses resultados.
\end{abstract}

Palavras-chave: Madeira da Amazônia, preservação, absorção, retenção e penetração.

\section{RETENTION ARIABILITY STUDY OF THE TIMBER PRESERVATIVE CCA TYPE A ON Brosimum rubescens Taub. MORACEAE - (PAU-RAINHA) WOOD}

\begin{abstract}
The variability of wood treatability of Brosimum rubescens Taub. Moraceae (pau-rainha) was evaluated. The tests were carried out on three different positions of the trunk (lower end, middle and upper end) and two parts (heartwood and sapwood).The process used was the Full-Cell, also known as "Bethell" process, with CCA-A, at $2 \%$ concentration. The analysis of variability was based on retention $\left(\mathrm{kg} / \mathrm{m}^{3}\right)$. Retention data from the different positions and parts of the trunk were compared. The data showed that the sapwood is moderately difficult to preserve, whereas the heartwood is refractory. Retention on different positions in the trunk did not present significant differences, but there is a significant difference in retention between heartwood and sapwood. Microdistribution analysis of wood preservative by means of wood anatomy is used as an auxiliary tool for the interpretation of the results.
\end{abstract}

Key words: $\quad$ Amazon wood, preservation, absorption, retention, and treatability.

\section{INTRODUÇÃO}

A preservação da madeira visa o uso adequado e, conseqüentemente, racional da floresta, uma vez que reduzirá bastante sua demanda, levando-se em consideração que uma espécie devidamente preservada terá sua vida útil prolongada.

A aplicação de um tratamento preservante só é economicamente viável se a vida útil da madeira tratada

1 Recebido para publicação em .

Aceito para publicação em .

2 Pesquisadores da Coordenação de Pesquisas de Produtos Florestais- Preservação da Madeira do Instituto Nacional de Pesquisas da Amazônia, Manaus Amazonas, Alameda Cosme Ferreira 1756, Aleixo, Caixa Postal 478, 69083-000 Manaus-AM, <ceci@ inpa.gov.br>. ${ }^{3}$ Professora Dra., Universidade do Amazonas, Departamento de Apoio à Pesquisa - PROPESP, Av. General Rodrigo Otávio Jordão Ramos, 3000, Aleixo, Manaus-AM. 
for significativamente aumentada em relação àquela usada sem tratamento.

Atualmente, dos métodos de preservação utilizados no mundo inteiro os mais eficientes são aqueles aplicados sob condições de vácuo e pressão, e dentre estes o mais importante é o de célula-cheia ou Bethell, que tem por fim preencher ao máximo as células da madeira com o preservativo (CTFT, 1970; Cockcroft, 1971; Déon, 1978).

A espécie em estudo é uma essência madeireira da região Amazônica, muito utilizada na movelaria decorativa, na confecção de cabos e objetos torneados, faqueados decorativos e de objetos de adorno, além de usos menos nobres, abrangendo a construção civil (INPACPPF,1991).

Através de pesquisas realizadas pela Coordenação de Pesquisas de Produtos Florestais, do Instituto Nacional de Pesquisas da Amazônia (INPA/CPPF, 1991), constatou-se que a espécie proposta no presente estudo apresenta boa trabalhabilidade. No entanto, nos ensaios de durabilidade natural em condições de campo, a espécie apresentou alta suscetibilidade a diversos Basidiomycetos (Jesus \& Bononi, 1991), o que sem dúvida compromete o seu valor comercial. Os testes de tratabilidade em condições de pressão revelaram boa capacidade de absorção para o alburno (INPA/CPPF, 1991). Isto evidencia a

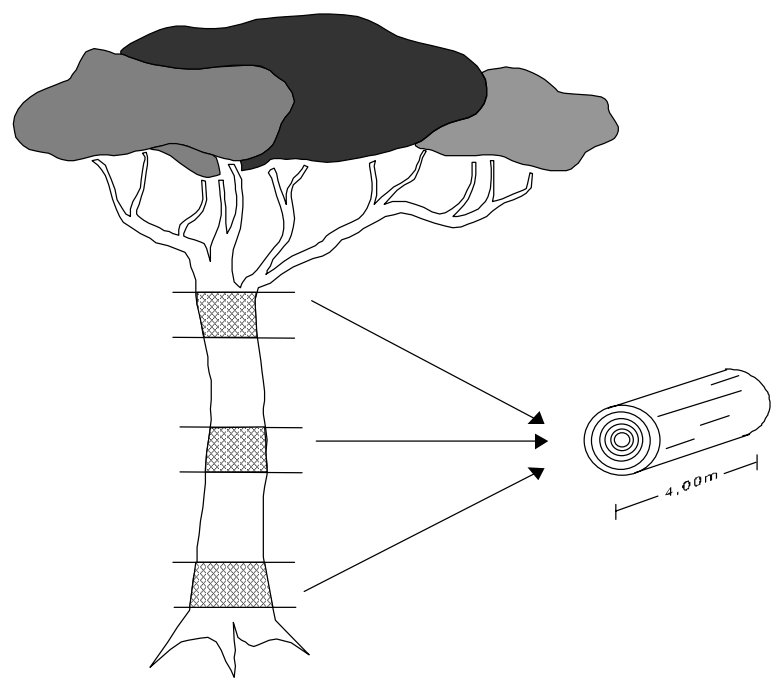

Figura 1 - Representação esquemática da amostragem. Figure 1 - Schematic representation of the sample.

R. Árvore, Viçosa-MG, v.27, n.6, p.845-853, 2003 importância da preservação, uma vez que sua finalidade é aumentar a vida útil de espécies madeireiras que apresentem baixa durabilidade natural, proporcionando-lhes maior resistência ao ataque de organismos xilófagos.

O objetivo deste traalho foi avaliar a tratabilidade de Brosimum rubescens Taub. Moraceae (pau-rainha), assim como a variação da tratabilidade ao longo de três alturas da árvore (base, meio e ápice) e entre duas partes (cerne e alburno), a fim de contribuir tecnologicamente para preservação da madeira, promovendo, assim, melhor aproveitamento da espécie.

\section{MATERIAL E MÉTODOS}

\subsection{Amostras}

Utilizaram-se três árvores, das quais foram retiradas três toras de $4 \mathrm{~m}$ de comprimento, selecionadas da base, do meio e do ápice. Os corpos-de-prova estudados tinham as seguintes características: foram obtidos do cerne e do alburno (Figura 1), obedecendo a um corte orientado nas seções transversal, tangencial e radial; passaram por um processo de secagem natural, até alcançar um teor médio de umidade de equilíbrio de $18 \%$; eram livres de defeitos; e possuíam dimensões finais de $5 \times 5 \times 70 \mathrm{~cm}$, conforme padrões IUFRO, representados por BECKER (1970) e AWPA-M7-83 (1989), com algumas adaptações.

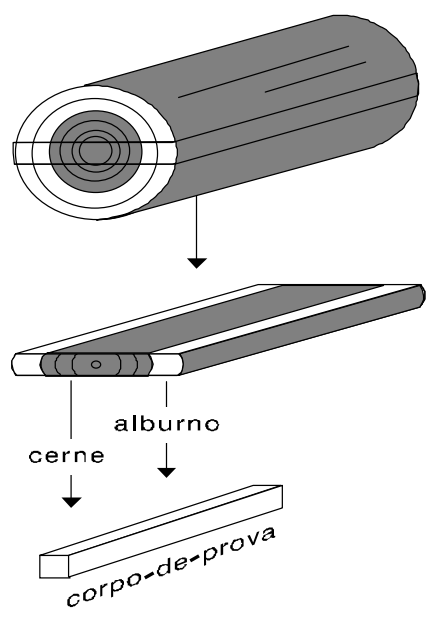


A seleção desses corpos-de-prova foi feita aleatoriamente, nas três toras. Suas extremidades foram vedadas com selador de pontas hidroasfáltico, para impedir a penetração pelos topos.

O material coletado é oriundo das proximidades do município de Presidente Figueiredo-Am, e o estudo compreendeu duas etapas: teste de tratabilidade da espécie, usando o método de célula-cheia (Bethell), e o estudo anatômico detalhado, abordando as estruturas anatômicas da espécie em relação à distribuição do preservante na madeira, o qual faz parte de outro trabalho, tendo sido enfocados os aspectos desta distribuição.

\subsection{Preservativo e Método de Impregnação}

O preservativo utilizado foi o arseniato de cobre cromatado (CCA), tipo A, a uma concentração de $2 \%$. O método adotado foi o célula-cheia ou Bethell (Figura 2), utilizado para tratabilidade em autoclave e aplicado sob condições de vácuo e pressão, no qual a madeira é tratada em condição seca (AFNOR, 1982). As etapas deste processo estão descritas na Figura 2.

\subsection{Critério de Avaliação do Grau de Tratabilidade}

Foi adotado o critério usado pelo Laboratório de Produtos Florestais LPF-Brasília e pelo Instituto de Pesquisas Tecnológicas do Estado de São Paulo (IPT-SP), com algumas modificações, conforme a proposta de um método simples para interpretação dos resultados de tratabilidade de Vianez \& Sales Neta (1989), utilizados para análise de preservação em autoclave com madeiras da Amazônia, testadas na CPPF (Sales Neta \& Vianez, 1991).

Terminado o processo de tratamento, os corpos-deprova foram retirados da autoclave e deixados em repouso para que o líquido excedente escorresse por um período de 30 minutos. Prosseguiu-se a avaliação da quantidade de líquido absorvido em cada peça, pela diferença de peso antes e após o tratamento.

Após a secagem natural sob abrigo, por um período de aproximadamente um mês, as peças foram seccionadas transversalmente a $20 \mathrm{~cm}$ de suas extremidades, e nas superfícies cortadas foi efetuado o teste de penetração

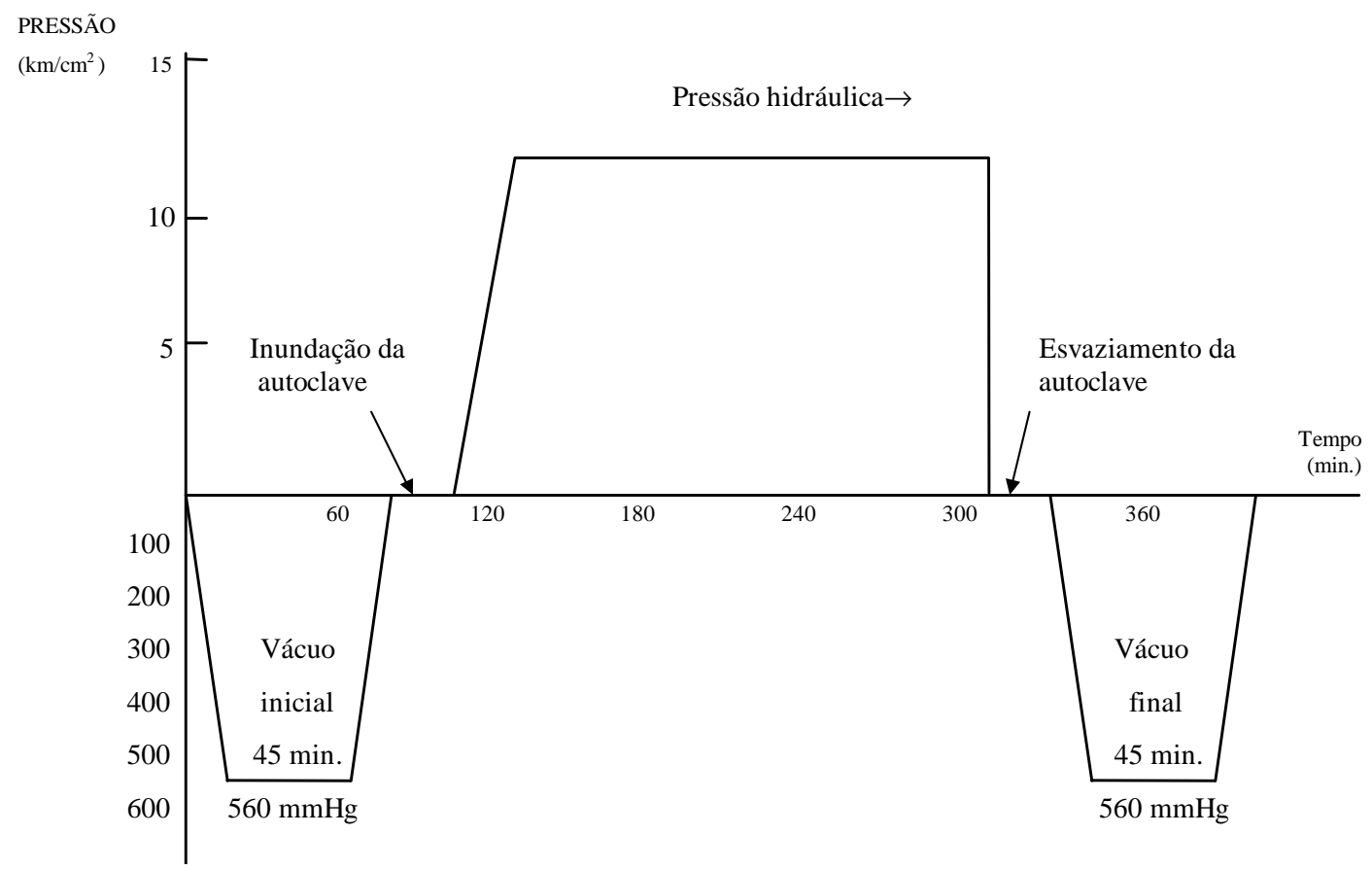

Figura 2 - Representação gráfica do processo de tratamento sob pressão "célula-cheia" utilizado nos testes de tratabilidade do INPA/CPPF.

Figure 2 - Graphic representation of the pressure treatment process "full cell" used in the tests of wood treatability by INPA/ $C P P F$. 
(colorimétrico), utilizando o Cromoazurol-S como indicador, pois ele reage com o preservante, mostrando uma coloração azulada, o que indica sua presença na madeira (IPT-DIMAD, 1980). A distribuição do preservante na peça foi classificada de acordo com os padrões mais comuns de penetração (IBDF/LPF 1988; Vianez \& Sales Neta 1989; INPA/CPPF, 1991), representados na Figura 3.

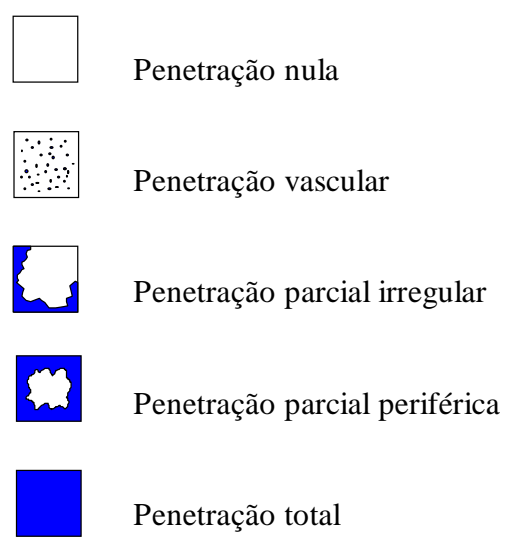

Figura 3 - Padrões mais comuns de penetração do teste de tratabilidade de madeira.

Figure 3-Most Common types of penetration in the wood treatability test

\subsection{Classificação da Tratabilidade}

A partir dos valores de absorção e dos tipos de penetração, a madeira foi classificada quanto à sua tratabilidade, obedecendo aos critérios de classificação apresentados no Quadro 1.

Foram feitos cortes histológicos, para observar a penetração do produto na madeira, abordando mais especificamente a área do alburno, uma vez que o cerne mostrou-se refratário quanto à sua tratabilidade, sendo este observado em nível de comparação. Os cortes foram imersos em Cromoazurol-S, para que a distribuição do preservante pudesse ser visualizada nas células da madeira.

Neste estudo, a variável testada para os três indivíduos de Brosimum rubescens foi a retenção, expressa $\mathrm{em} \mathrm{kg} / \mathrm{m}^{3}$, tendo sido observadas três posições (base, meio e ápice) e duas partes (cerne e alburno), com o fim de verificar diferenças de retenções entre os fatores preestabelecidos no experimento.

R. Árvore, Viçosa-MG, v.27, n.6, p.845-853, 2003
A escolha da variável retenção para a análise estatística, em vez de absorção, deu-se em função de a primeira expressar o que ficou realmente retido na madeira após o processo de absorção e evaporação da água, ou seja, os ingredientes ativos contidos no preservante, sendo a resultante da absorção expressa em $\mathrm{kg} / \mathrm{m}^{3}$, enquanto a absorção é expressa em 1/ $1 \mathrm{~m}^{3}$, sendo uma proporcional à outra.

Os dados do experimento foram considerados inteiramente casualizados no esquema fatorial $3^{2} \times 2$, para testar os efeitos dos fatores escolhidos, em três exemplares da espécie, tendo sido considerados três corposde-prova por posição (base/meio/ápice), em cada parte (cerne/alburno), para cada indivíduo do presente estudo.

\section{RESULTADOS E DISCUSSÃO}

Os valores médios de absorção e retenção encontram-se no Quadro 2, assim como o tipo de penetração, o grupo de absorção e a classificação quanto à tratabilidade para as três alturas da árvore e as diferentes partes estudadas (cerne e alburno), os quais foram obtidos a partir da classificação do Quadro 1.

Os resultados estatísticos mostram que houve diferença significativa de retenção $(\mathrm{P}<0,05)$ apenas para o efeito parte (cerne e alburno), enquanto o fator altura (base, meio e ápice) não apresentou diferença significativa entre os níveis deste fator (Quadro 3, Figura 4).

O alburno, por pertencer ao grupo 3 de absorção em todas as alturas (base, meio e ápice), por variar de 397,44 a $442,141 / \mathrm{m}^{3}$ e ter um padrão de penetração do tipo parcial periférica, classifica-se, quanto à sua tratabilidade, como madeira moderadamente difícil de tratar. Tal resultado difere dos encontrados em estudos de tratabilidade efetuados anteriormente com a mesma espécie (INPA/CPPF, 1991), nos quais o alburno foi classificado como madeira moderadamente fácil de tratar.

É provável que as condições de microclima ou mesmo as possíveis diferenças de idades das árvores tenham contribuído para os resultados diferentes daqueles anteriores, principalmente sabendo-se que árvores mais velhas apresentam maiores deposições de extrativos, obstruindo os vasos e os raios, o que, aliás, foi observado no presente estudo.

O cerne, por possuir penetração nula em todas as alturas e valores muito baixos de absorção (de 6,66 a $\left.13,26 \mathrm{l} / \mathrm{m}^{3}\right)$, é considerado refratário quanto ao processo de impregnação em questão, comportando-se de maneira semelhante ao do estudo anterior (INPA/CPPF, 1991). 
Quadro 1 - Modelo usado para classificação da tratabilidade da madeira pelo INPA/CPPF Tabela 1 - Model used for classification of wood treatability by INPA/CPPF

\begin{tabular}{|c|c|c|c|}
\hline Absorção (Valor Numérico) & Penetração (Valor Numérico) & Absorção X Penetração & Tratabilidade \\
\hline $\begin{array}{cc}0 \mid------150 & 1 / \mathrm{m}^{3} \\
\text { Grupo } & 1(0,00) \\
\text { “ } & \text { “ } \\
\text { “ } & \text { ، } \\
\text { “ } & \text { “ } \\
\text { “ } & \text { “ }\end{array}$ & $\begin{array}{ll}\mathrm{N} & (0,00) \\
\mathrm{V} & (0,25) \\
\mathrm{PI} & (0,50) \\
\mathrm{PP} & (0,75) \\
\mathrm{T} & (1,00)\end{array}$ & $\begin{array}{l}0,00 \\
0,00 \\
0,00 \\
0,00 \\
0,00\end{array}$ & $\begin{array}{l}\mathrm{R} \\
\mathrm{R} \\
\mathrm{R} \\
\mathrm{R} \\
\mathrm{X}\end{array}$ \\
\hline $\begin{array}{cc}\text { 150|------300 } & 1 / \mathrm{m}^{3} \\
\text { Grupo } & 2(0,25) \\
\text { “ } & \text { ، } \\
\text { ، } & \text { ، } \\
\text { ، } & \text { ، } \\
\end{array}$ & $\begin{array}{ll}\mathrm{N} & (0,00) \\
\mathrm{V} & (0,25) \\
\mathrm{PI} & (0,50) \\
\mathrm{PP} & (0,75) \\
\mathrm{T} & (1,00) \\
\end{array}$ & $\begin{array}{l}0,00 \\
0,06 \\
0,12 \\
0,19 \\
0,25\end{array}$ & $\begin{array}{l}\mathrm{R} \\
\mathrm{D} \\
\mathrm{D} \\
\mathrm{D} \\
\mathrm{D}\end{array}$ \\
\hline $\begin{array}{cc}300 \mid-----450 & 1 / \mathrm{m}^{3} \\
\text { Grupo } & (0,50) \\
\text { ، } & \text { ، } \\
\text { ، } & \text { ، } \\
\text { ، } & \text { ، } \\
\text { “ } & \text { ، }\end{array}$ & $\begin{array}{ll}\mathrm{N} & (0,00) \\
\mathrm{V} & (0,25) \\
\mathrm{PI} & (0,50) \\
\mathrm{PP} & (0,75) \\
\mathrm{T} & (1,00)\end{array}$ & $\begin{array}{l}0,00 \\
0,12 \\
0,25 \\
0,38 \\
0,50\end{array}$ & $\begin{array}{c}\mathrm{X} \\
\mathrm{D} \\
\mathrm{D} \\
\mathrm{MD} \\
\mathrm{MD}\end{array}$ \\
\hline $\begin{array}{c}\text { 450|-----600 } 1 / \mathrm{m}^{3} \\
\text { Grupo } 4(0,75) \\
\text { “، } \\
\text { “، } \\
\text { ، “ } \\
\text { ، “ } \\
\text { ، “ } \\
\end{array}$ & $\begin{array}{ll}\mathrm{N} & (0,00) \\
\mathrm{V} & (0,25) \\
\mathrm{PI} & (0,50) \\
\mathrm{PP} & (0,75) \\
\mathrm{T} & (1,00) \\
\end{array}$ & $\begin{array}{l}0,00 \\
0,19 \\
0,38 \\
0,56 \\
0,75\end{array}$ & $\begin{array}{c}\mathrm{X} \\
\mathrm{D} \\
\mathrm{MD} \\
\mathrm{MD} \\
\mathrm{MF}\end{array}$ \\
\hline 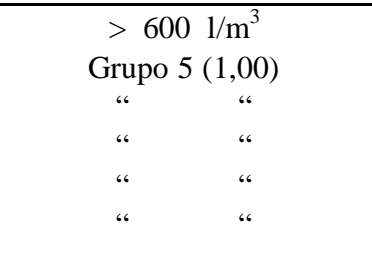 & $\begin{array}{ll}\mathrm{N} & (0,00) \\
\mathrm{V} & (0,25) \\
\mathrm{PI} & (0,50) \\
\mathrm{PP} & (0,75) \\
\mathrm{T} & (1,00)\end{array}$ & $\begin{array}{l}0,00 \\
0,25 \\
0,50 \\
0,75 \\
1,00\end{array}$ & $\begin{array}{c}\mathrm{X} \\
\mathrm{X} \\
\mathrm{MD} \\
\mathrm{MF} \\
\mathrm{F}\end{array}$ \\
\hline
\end{tabular}

$\mathrm{N}=$ nula, $\mathrm{V}=$ vascular, $\mathrm{P} \mathrm{I}=$ parcial irregular, $\mathrm{PP}=$ parcial periférica, $\mathrm{T}=$ total, $\mathrm{R}=$ refratária, $\mathrm{X}=$ não ocorre na prática, $\mathrm{D}=$ difícil, $\mathrm{F}=$ fácil, $\mathrm{MF}=$ moderadamente fácil e $\mathrm{MD}=$ moderadamente difícil .

Fonte: Vianez \& Sales Neta (1989).

É notória a diferença da retenção entre o cerne e o alburno. Tal fato relaciona-se às características anatômicas destas estruturas, cujas maiores deposições de extrativos encontram-se presentes no cerne, bem como as maiores obstruções dos vasos deste por tiloses (Figura 5B-D).

Em uma análise microscópica da distribuição das estruturas anatômicas do cerne e do alburno, através de cortes histológicos de amostras preservadas, observouse grande quantidade de extrativos presentes nos raios do cerne, como também em diversos raios do alburno, assim como no parênquima axial e em pontuações vasculares (Figura 5), dificultando a distribuição do preservante na madeira.

$\mathrm{O}$ raio e o parênquima axial não contribuíram de maneira efetiva para a dispersão do preservante (Figura 5A-B), divergindo de Wardrop \& Davies (1961) e de Siau (1971), quando enfatizam os raios como importantes vias de penetração em uma representação esquemática da fluência de líquido nas folhosas. Neste trabalho, ao

R. Árvore, Viçosa-MG, v.27, n.6, p.845-853, 2003 
Quadro 2 - Classificação da tratabilidade da madeira nas árvores estudadas Table 2 - Wood treatability classification for the studied trees

\begin{tabular}{|c|c|c|c|c|c|c|c|}
\hline Árvore & Altura & Parte & $\begin{array}{c}\text { Absorção Média } \\
\left(1 / \mathrm{m}^{3}\right)\end{array}$ & $\begin{array}{c}\text { Retenção Média } \pm \\
\text { Desvio-Padrão }\left(\mathrm{kg} / \mathrm{m}^{3}\right)\end{array}$ & Penetração & Grupo & Classificação \\
\hline \multirow{6}{*}{1} & \multirow{2}{*}{ base } & cerne & 8,93 & $0,18 \pm 0,01$ & $\mathrm{~N}$ & 1 & $\mathrm{R}$ \\
\hline & & alburno & 401,56 & $8,03 \pm 0,07$ & $\mathrm{PP}$ & 3 & $\mathrm{MD}$ \\
\hline & \multirow{2}{*}{ meio } & cerne & 9,83 & $0,20 \pm 0,01$ & $\mathrm{~N}$ & 1 & $\mathrm{R}$ \\
\hline & & alburno & 412,71 & $8,25 \pm 0,03$ & PP & 3 & $\mathrm{MD}$ \\
\hline & \multirow{2}{*}{ ápice } & cerne & 13,26 & $0,27 \pm 0,04$ & $\mathrm{~N}$ & 1 & $\mathrm{R}$ \\
\hline & & alburno & 397,44 & $7,95 \pm 0,88$ & PP & 3 & $\mathrm{MD}$ \\
\hline \multirow{6}{*}{2} & \multirow{2}{*}{ base } & cerne & 6,66 & $0,13 \pm 0,02$ & $\mathrm{~N}$ & 1 & $\mathrm{R}$ \\
\hline & & alburno & 405,38 & $8,11 \pm 0,26$ & PP & 3 & $\mathrm{MD}$ \\
\hline & \multirow{2}{*}{ meio } & cerne & 10,19 & $0,20 \pm 0,03$ & $\mathrm{~N}$ & 1 & $\mathrm{R}$ \\
\hline & & alburno & 414,61 & $8,29 \pm 0,31$ & PP & 3 & MD \\
\hline & \multirow{2}{*}{ ápice } & cerne & 9,04 & $0,18 \pm 0,04$ & $\mathrm{~N}$ & 1 & $\mathrm{R}$ \\
\hline & & alburno & 430,71 & $8,61 \pm 0,23$ & PP & 3 & $\mathrm{MD}$ \\
\hline \multirow{6}{*}{3} & \multirow{2}{*}{ base } & cerne & 7,48 & $0,15 \pm 0,01$ & $\mathrm{~N}$ & 1 & $\mathrm{R}$ \\
\hline & & 'alburno & 442,14 & $8,84 \pm 0,36$ & PP & 3 & MD \\
\hline & \multirow{2}{*}{ meio } & cerne & 10,89 & $0,22 \pm 0,06$ & $\mathrm{~N}$ & 1 & $\mathrm{R}$ \\
\hline & & alburno & 401,52 & $8,03 \pm 0,07$ & PP & 3 & MD \\
\hline & \multirow{2}{*}{ ápice } & cerne & 12,54 & $0,25 \pm 0,05$ & $\mathrm{~N}$ & 1 & $\mathrm{R}$ \\
\hline & & alburno & 419,35 & $8,39 \pm 0,47$ & PP & 3 & MD \\
\hline
\end{tabular}

$\mathrm{R}=$ refratária, $\mathrm{MD}=$ moderadamente difícil, $\mathrm{N}=$ nula e $\mathrm{PP}=$ parcial periférica.

$R=$ refractory, $M D=$ moderately difficult, $N=$ null and $P P=$ partially peripheral .

Quadro 3 - Análise de variância da retenção $\left(\mathrm{kg} / \mathrm{m}^{3}\right)$ de acordo com o esquema fatorial $3^{2} \times 2$ Table 3 - Variance analysis of retention $\left(\mathrm{kg} / \mathrm{m}^{3}\right)$ according to factorial scheme $3^{2} \times 2$

\begin{tabular}{|c|c|c|c|c|c|}
\hline Fonte de Variação & GL & SQ & Q.M & $\mathrm{F}$ & $\operatorname{Pr}>\mathrm{F}$ \\
\hline Árvore & 2 & 0,2597 & 0,12985 & 1,72 & 0,19290 \\
\hline Posição & 2 & 0,0512 & 0,02560 & 0,34 & 0,71400 \\
\hline Parte & 1 & 881,6423 & 881,64230 & $1.1700,15$ & $0,00018 *$ signif. \\
\hline Árvore *Posição & 4 & 0,6524 & 0,16310 & 2,16 & 0,09280 \\
\hline Árvore *Parte & 2 & 0,3225 & 0,16125 & 2,14 & 0,13240 \\
\hline Posição *Parte & 2 & 0,0789 & 0,03945 & 0,52 & 0,59700 \\
\hline Árvore *Posição *Parte & 4 & 0,7926 & 0,19815 & 2,63 & 0,05030 \\
\hline Resíduo & 36 & 2,7127 & 0,07530 & - & \\
\hline Total & 53 & 886,5124 & - & - & \\
\hline
\end{tabular}

contrário do observado pelos autores citados, o raio mostrou-se ineficiente quanto à condução do preservante na madeira. Os resultados mostram a má condução dos raios no presente estudo, o que concorda com Hunt \& Garrat (1953) e Behr et al. (1969), quando relataram a variabilidade destas estruturas.

R. Árvore, Viçosa-MG, v.27, n.6, p.845-853, 2003
Foram observadas, principalmente, obstruções dos vasos por tiloses no cerne e em diversas regiões do alburno (Figura 5B-D), as quais contribuíram de maneira relevante para a má dispersão do preservante na madeira. Embora os vasos sejam considerados estruturas de maior importância em relação à condução (Hunt \& Garrat, 1953; 
Wardrop \& Davies, 1961; Rudman, 1965; Behr et al., 1969; Greaves, 1972, 1973), eles não contribuíram de maneira eficiente para a condução do preservante na espécie estudada, não só em função das tiloses, como também devido a freqüentes obstruções das pontuações vasculares por extrativos, encontradas nas paredes dos mesmos, conforme observa-se na Figura 4A; E. Tais observações coincidem com as de Côté (1963), que, ao analisar as características anatômicas que afetavam a permeabilidade da madeira, encontrou como uma das causas a oclusão das pontuações por extrativos.

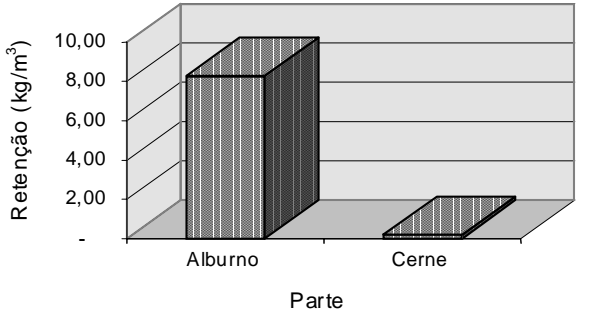

Figura 4 - Retenção média do CCA por partes da madeira cerne e alburno $\left(\mathrm{kg} / \mathrm{m}^{3}\right)$.

Figure 4 - Average retention of CCA in heartwood and $\operatorname{sapwood}\left(\mathrm{kg} / \mathrm{m}^{3}\right)$.

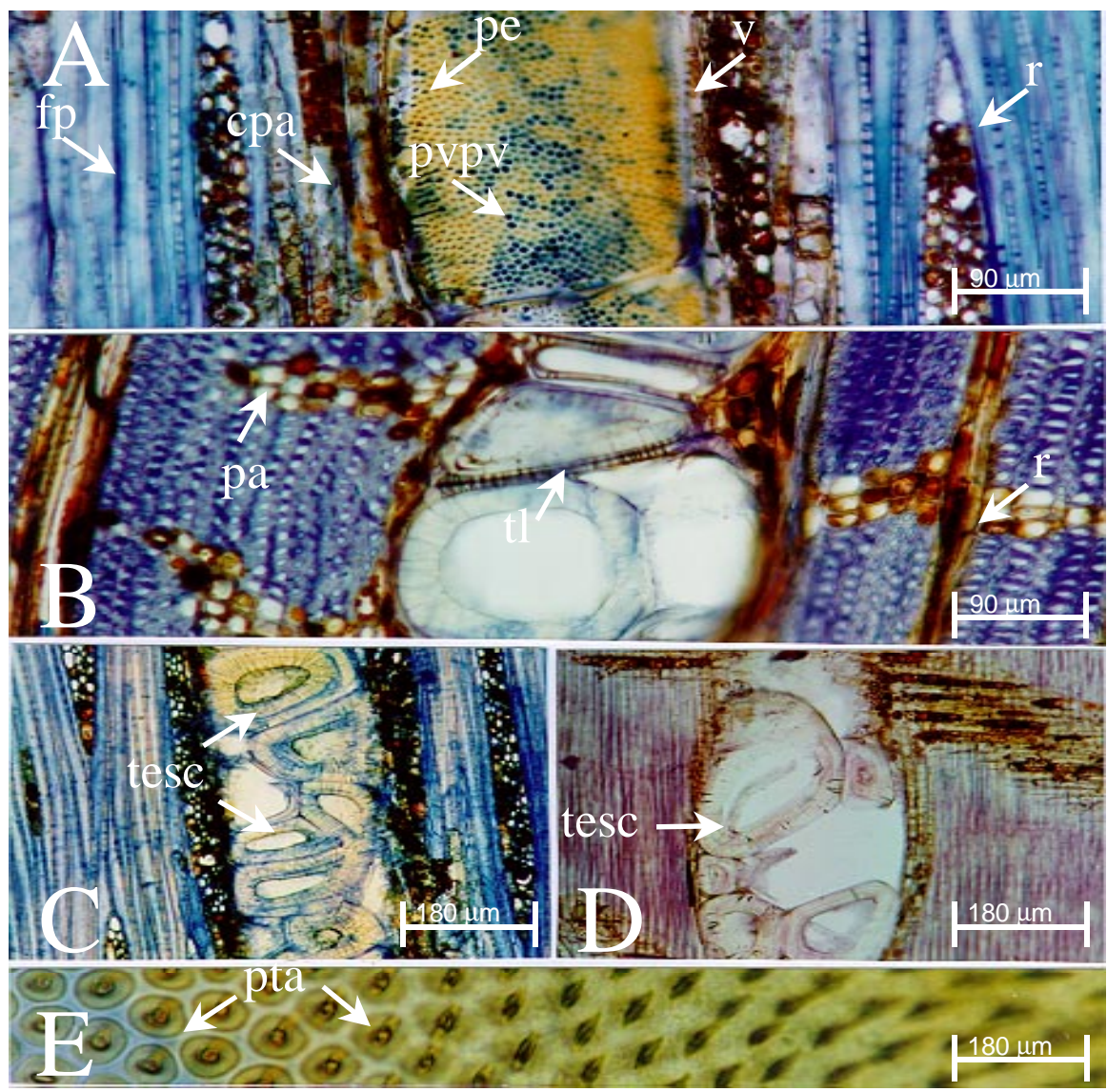

$\mathrm{A}=$ corte tangencial externo, $\mathrm{B}=$ transversal, $\mathrm{C}=$ tangencial externo, $\mathrm{D}=$ radial interno, $\mathrm{E}=$ detalhe das pontuações vasculares, $\mathrm{fp}=$ fibras preservadas, $\mathrm{cpa}=$ células do parênquima axial, $\mathrm{pe}=$ pontuações com extrativos, $\mathrm{pvpv}=$ pontuações vasculares com preservante, $\mathrm{v}=\mathrm{vaso}, \mathrm{r}=$ raio, $\mathrm{pa}=$ parênquima axial, $\mathrm{tl}=$ tilose, tesc $=$ tilose esclerificada e $\mathrm{pta}=$ pontuações areoladas.

$A=$ outer tangential section, $B=$ cross section, $C=$ outer tangential section, $D=$ inner radial section, $E=$ details of vessel pits, $F p=$ preserved fibres, cpa = axial parenchyma cells, pe = pits with extractives, $p v p v=$ vessel pits with preservative, $v=v e s s e l, r=$ ray, pa $=$ axial parenchyma, $t l=$ tyosis, tesc $=$ sclerosed tylosis and pta= bordered pits.

Figura 5 - Microdistribuição do CCA na madeira de Brosimum rubescens.

Figure 5 - Microdistribution of CCA in Brosimum rubescens wood. 
Greaves (1974) relatou que a penetração nas fibras parece ser extremamente variável. $\mathrm{O}$ atual estudo evidencia a importância da estrutura, onde sua relevância pode ser observada (Figura 5A), o que concorda com Hunt \& Garrat (1953) e discorda de Stone \& Green (1958), que acreditavam que a fibra contribuía muito pouco ou quase nada para a penetração de líquido na madeira. Com relação a este fato, Hunt \& Garrat (1953) relataram que as fibras assumem importante papel na dispersão do preservante, tornando-se às vezes mais importantes do que os vasos, quando estes apresentam obstruções por tiloses, embora eles sejam normalmente os elementos responsáveis pela maior condução do preservante, fato também observado neste estudo.

Questões como localização e retenção do preservante nas células da madeira são fundamentais na demonstração da eficácia do método de tratamento e na ação do preservante em relação à resistência à decomposição por organismos xilófagos (BEHR et al.,1969).

No presente estudo pôde-se observar que a anatomia da madeira é de fundamental importância para melhor compreensão dos resultados de tratabilidade, fornecendo ferramentas para sua interpretação e promovendo subsídios que permitam auxiliar a indicação de um tratamento adequado.

Quanto à tratabilidade, observou-se que a espécie em estudo apresenta cerne refratário e alburno moderadamente difícil de tratar, tendo sido utilizado o mesmo processo de impregnação do estudo anterior (INPA/CPPF, 1991). Constatou-se que a maioria das espécies madeireiras da Amazônia é de difícil tratabilidade, o que concorda com vários estudos realizados com tais espécies (IBDF/LPF, 1981, 1988; INPA/CPPF, 1991, 1993).

A alternativa para estas espécies de difícil tratabilidade possivelmente seria o tratamento por difusão. O método oferece a vantagem de impregnação através dos capilares da parede celular, penetrando, portando, mais profundamente. Através da difusão pode-se obter uma boa penetração, mesmo em espécies refratárias (Richardson, 1978; Wilkinson, 1979).

\section{CONCLUSÕES}

- O cerne é considerado refratário e o alburno, moderadamente difícil de preservar.

- O alburno apresentou nível de absorção e retenção significativamente maior que o do cerne.

R. Árvore, Viçosa-MG, v.27, n.6, p.845-853, 2003
- Não houve diferença significativa de absorção e retenção do produto em relação às alturas da árvore (base, meio e ápice), tanto para o cerne quanto para o alburno.

- A obstrução dos vasos do cerne por tiloses contribuiu sensivelmente para o caráter refratário do cerne, bem como para a permeabilidade limitada do alburno.

- As deposições de extrativos presentes no raio contribuíram para a má condução do preservante nestas células e, conseqüentemente, para a má dispersão do preservante na madeira.

\section{REFERÊNCIAS BIBLIOGRÁFICAS}

AFNOR Recueil de normes françaises Paris - La Défense, 1982. 514 p.

AMERICAN WOOD PRESERVERS ASSOCIATION. Standard method of evaluating wood preservatives by field tests with stakes M7 - 83. 1989. 7 p.

BECKER, G. Método padrão sugerido pela IUFRO para ensaios de campo com estaca de madeira. Preservação da madeira, v. 1, n. 4, p. 205-216, 1970.

BEHR, E. A. et al. Microscopic examination of pressuretreated wood. Forest Production Journal, v. 19, n. 8, p. 31-41, 1969.

CENTRE TECHNIQUE FORESTIER TROPICAL DIVISION DE PRÉSERVATION. Note sur l'injection des bois en autoclave. France: 1970.7 p.

COCKCROFT, R. Timbers preservatives and methods of treatment. Timberlab Papers Princes Risborough Laboratory, v. 46, p. 1-6, 1971.

CÔTÉ, W. Structural factors affecting the permeability of wood. Journal Polymer Science, v. 2, p. 231-242, 1963.

DÉON, G. Manuel de préservation des bois en climat tropicaux. Nogent-Sur-Marne France: CTFT 1978. 111 p.

GREAVES, H. Structural distribution of chemical components in preservative treated wood by energy dispersion X- Ray analyses. Mat. U. Organismen, v. 7, p. 277-285, 1972.

GREAVES, H. X-ray analyses of selected anatomical structure in Copper-Chrome-Arsenic treated wood. Finland: International Research Group on Wood Press. 1973. (IRG/ WP320).

GREAVES, H. A review of the influence of structural anatomy on liquid penetration into hardwoods. Victoria: C.S.I.R.O. Forest Production Laboratory, 1974. p 37-40. 
HUNT, G. M.; GARRATT, G. A. Wood preservation. 2.ed. New York: McGraw Hill Book Co. 1953. 417 p.

INSTITUTO BRASILEIRO DE DESENVOLVIMENTO FLORESTAL/LABORATÓRIO DE PRODUTOS FLORESTAIS - IBDF/LPF. Madeiras da Amazônia; Características e utilização -Floresta Nacional do Tapajós. Brasília: CNPq. 1981. v.1. 113 p.

INSTITUTO BRASILEIRO DE DESENVOLVIMENTO FLORESTAL / LABORATÓRIO DE PRODUTOS FLORESTAIS - IBDF/LPF. Madeiras da Amazônia, Características e utilização. Estação Experimental de Curuá-Una, v. 2, p. 30-32, 1988.

INSTITUTO NACIONAL DE PESQUISAS DA AMAZÔNIA / CENTRO DE PESQUISA DE PRODUTOS FLORESTAIS - INPA/CPPF. Catálogo de madeiras da Amazônia: características tecnológicas; área da hidrelétrica de Balbina. Manaus: 1991. 163 p.

INSTITUTO NACIONAL DE PESQUISAS DA AMAZÔNIA / CENTRO DE PESQUISA DE PRODUTOS FLORESTAIS - INPA/CPPF. Catalogo de madeiras da Amazônia: Características Tecnológicas. Manaus: 1993. $58 \mathrm{p}$.

IPT-DIMAD Reações colorimétricas para determinação de preservativo em madeiras-DIMAD 1980. C1. In: Métodos de ensaio e análises em preservação de madeiras Public 1155. 1980.

JESUS, M. A.; BONONI, V. Fungos em essências florestais da área da usina hidrelétrica de Balbina, Presidente Figueiredo, Am. ABPM, v. 70, p. 19, 1991.
PRESERVA PRODUTOS QUÍMICOS LTDA. Manual de tratamento de madeiras. São Paulo, 1980. 55 p.

RICHARDSON, B. A. Wood preservation. Lancaster: The Construction Press. 1978. 238 p.

RUDMAN, P. Fine structure of wood. Nature, v. 208, p. 55-56, 1965.

SALES NETA, C.; VIANEZ, B. F. Chemical treatment of ten Amazônia timber species of low natural durability. In: ANNUAL MEETING, Japan Doc. 1991. 14 p. (IRG/WP/ 3640).

SIAU, J. F. Flow in wood. New York: Syracuse University Press, 1971. $131 \mathrm{p}$.

STONE, J. E.; GREEN, H. V. Penetration and diffusion into hardwoods. Pulp and Paper Mag., v. 50, p. 223-232, 1958.

VIANEZ, B. F.; SALES NETA, C. Proposta de um método simples para interpretação dos resultados de tratabilidade de madeiras. In: ENCONTRO BRASILEIRO EM PRESERVAÇÃO DE MADEIRAS, 3., 1989, São Paulo. Anais... São Paulo: 1989. 9 p.

WARDROP, A. B.; DAVIES G. W. Morphological factors relating to the penetration of liquids into wood. Holzforschung, v. 15, n. 5, p.129-141, 1961.

WILKINSON, J. G. Industrial timber preservation. London: Rentokil Ltd. Assoc. Business Press, 1979. $532 \mathrm{p}$. 